INTERNACIONAL

\title{
Nuevas fórmulas judiciales para enfrentar casos de vulneración de derechos humanos por organizaciones internacionales
}

\author{
New judicial formulas to face cases of vulneration of human rights \\ by international organizations
}

\section{Gloria Ramos-Fuentes}

Universidad Autónoma de Chile

Patricio Masbernat

Universidad Autónoma de Chile

\begin{abstract}
RESUMEN La inmunidad de jurisdicción de las organizaciones internacionales se ha basado en diversas teorías, que generalmente se agrupan en tesis absoluta, relativa y funcional. Estas teorías no han sido suficientes para responder a los problemas judiciales actuales en los que las organizaciones internacionales han estado involucradas en varios países del mundo. Como resultado de esto, varios especialistas de gran prestigio han entregado ideas para enfrentar estos nuevos problemas. Además, diversos tribunales han estado construyendo nuevas tesis que responden a la realidad internacional actual en la que estas organizaciones se mueven. Hoy podría ser factible construir una nueva tesis desde la perspectiva de la doctrina legal del derecho internacional público. En efecto, algunos tribunales internacionales y nacionales de diferentes países han enfrentado este tema desde una perspectiva diferente, según una teoría que podría llamarse el principio de quid pro quo (o reciprocidad y equivalencia). Los autores de este artículo buscan mostrar estas nuevas tendencias judiciales y razonar sobre ellas.
\end{abstract}

PALABRAS CLAVE Inmunidad de jurisdicción, organizaciones internacionales, derechos humanos; decisiones judiciales.

ABSTRACT The immunity of jurisdiction of international organizations has been based on various theories of Law, which are usually grouped in the absolute thesis, the relative thesis and the functional thesis. These theories have not been enough to respond to the current judicial problems in which international organizations have been involved in various countries. As a result of this, several specialists of great prestige have delivered 
ideas to face these problems. Also, various courts have been building new theses that respond to the current international reality in which these organizations are moving. Today it might be possible to build a new thesis from the perspective of the legal doctrine of Public International Law. Indeed, some international and national courts of different countries have faced this issue from a different perspective, according to a theory that could be called the principle of quid pro quo (or reciprocity and equivalence). The authors of this paper seek to show these new judicial tendencies and reason about them.

KEYWORDS Immunity of jurisdiction, international organizations, human rights, judicial decisions.

\section{Introducción}

Las organizaciones internacionales tienen por objeto colaborar en coordinar esfuerzos de paz y desarrollo para diversos países e incluso de manera universal. Constituyen sujetos de derecho internacional, dotados de privilegios e inmunidades para efectuar sus funciones sin obstáculos de los Estados en que ellas operan. Sin embargo, en un mundo globalizado y complejo, en que las actividades de las organizaciones internacionales han aumentado en número e intensidad, se pueden generar situaciones no previstas en un inicio. Unas de ellas pueden ser los atentados a derechos humanos por parte de estas organizaciones. El problema es cómo se solucionan estos casos, en la medida en que gozan de importantes inmunidades. Este es el problema particular intenta responder este artículo.

En primer término, hay que considerar que el origen y los fundamentos de la inmunidad de jurisdicción de los Estados presenta diferentes particularidades que la inmunidad de jurisdicción de las organizaciones (De Brabandere, 2010: 83). Así, si bien es cierto que comparten contenidos y aspectos similares, las inmunidades de las organizaciones poseen características autónomas, tanto por su origen como por su fundamento y alcance, sin perjuicio de lo cual comparten el reto de responder de manera adecuada a las exigencias del derecho internacional en general - sobre todo, como se mostrará, el principio de buena fe-, y del respeto a los derechos humanos en particular (Espósito, 2007).

Tradicionalmente, respecto de las organizaciones internacionales se han defendido las doctrinas de la inmunidad jurisdiccional absoluta, relativa y funcional, con esta última como la que posee más adherentes en la doctrina. En los últimos años, y en búsqueda de respuestas a la insuficiencia de las teorías señaladas (pues no resuelven todo el problema generado con la inmunidad de las organizaciones internacionales), importantes autores de derecho internacional han levantado fundamentos para otra doctrina que complementa a la funcional, que se ha denominado por algunos como quid pro quo, y que incorpora por una parte la idea de reciprocidad y, por otra, 
el principio de buena fe que debe imperar en las relaciones entre los sujetos de derecho internacional. Este criterio se encuentra en desarrollo, y si bien ha sido incorporado en algunas sentencias de tribunales nacionales e internacionales y por algunos importantes juristas, no ha sido aún completa y uniformemente delineado.

\section{Primeras aproximaciones}

Una primera aproximación del principio quid pro quo como fórmula para ser aplicada a los casos conflictivos de inmunidad de jurisdicción de las organizaciones internacionales y derechos humanos, es posible encontrarla en la opinión consultiva de la Corte Internacional de Justicia de 1954, sobre «Effect of awards of compensation made by the UN Administrative Tribunal», en la que manifestó que la creación del Tribunal Administrativo de Naciones Unidas era adecuada a los fines de "promover libertad y justicia para los individuos» (Al Jaghoub, 2006).

Al respecto, la Asamblea General de Naciones Unidas consultó a la Corte si tenía el derecho a negarse a dar efecto a una sentencia de su Tribunal Administrativo, que otorgaba una indemnización a favor de un miembro de su personal cuyo contrato de servicio había sido rescindido sin su consentimiento. A continuación, y en caso de que la respuesta fuera afirmativa, la Asamblea General requirió a la Corte un pronunciamiento acerca de cuáles serían los principales motivos por los cuales podría ejercer legalmente ese derecho.

Para efectos de contestar la primera consulta, la Corte consideró que debía establecerse, en primer término, si la Asamblea General se encontraba investida, según la Carta de Naciones Unidas, de los poderes legales para crear un tribunal competente para emitir sentencias vinculantes para dicha organización. Al respecto, señaló que no existe en la mencionada Carta ninguna disposición expresa que permita el establecimiento de cuerpos u órganos judiciales, y a la vez no existe ninguna indicación en contrario. Para resolver este tema sin norma expresa que lo regule, la Corte recurrió a su opinión en «Reparation for injuries suffered in the service of the United Nations, Advisory», ${ }^{2}$ en la cual aplica la teoría de la existencia de poderes implícitos de Naciones Unidas.

La Corte se preguntó, entonces, si las disposiciones de la Carta relativas a las relaciones entre los miembros del personal y la organización implicaban para la organización el poder de establecer un tribunal judicial para conocer de las controversias que surjan de los contratos de servicio. ${ }^{3}$ La Corte desarrolló dos razonamientos:

1. «Effect of awards of compensation made by the UN Administrative Tribunal», opinión asesora de la Corte Internacional de Justicia, 13 de julio de 1954, p. 47, disponible en https://bit.ly/2MDlqlV.

2. «Reparation for injuries suffered in the service of the United Nations», opinión asesora de la Corte Internacional de Justicia, 1949, p. 182.

3. «Effect of awards», p. 56. 
- En primer término, analizó el objetivo de la Carta de la ONU, lo que la llevó a reflexionar de la siguiente manera: «En opinión de la Corte, difícilmente sería coherente con el objetivo expreso de la Carta de promover la libertad y la justicia para los individuos y con la constante preocupación de la Organización de las Naciones Unidas de promover este objetivo, que no se permita ningún recurso judicial o arbitral para su propio personal para la solución de cualquier disputa que pueda surgir entre esta y ellos» (p. 57; la traducción es nuestra).

- Además, en consideración a lo anterior, sostiene que la facultad de establecer un tribunal que haga justicia entre Naciones Unidas y su personal es necesaria, según lo dispuesto en la Carta, para asegurar los más altos estándares de eficiencia, competencia e integridad.

Si bien es cierto que esta opinión se pronuncia sobre la creación de un Tribunal Administrativo de Naciones Unidas, es decir, de carácter interno que resolverá conflictos entre ésta y sus funcionarios, lo relevante es que reconoce la existencia de a lo menos un compromiso por parte de la organización, que hoy podríamos configurar como una obligación jurídica evidente de resolver los conflictos que se le presenten, ya sea con sus funcionarios o terceros, a partir de la noción de derechos humanos (acceso a la justicia), y también de Estado de derecho, cuya fuente es el derecho internacional consuetudinario y el convencional. ${ }^{4}$ Según es posible observar en pronunciamientos de diversos países, y desde una perspectiva general de la doctrina, esos fundamentos son posibles de trasladar y aplicar a los conflictos generados entre una organización internacional y terceros (personas naturales) dentro de la jurisdicción de un Estado.

\section{Tribunal Europeo de Derechos Humanos}

Un desarrollo más exhaustivo sobre la materia se encuentra en las sentencias del Tribunal Europeo de Derechos Humanos, en los casos Wait \& Kennedy con Alemania5 y Beer \& Regan con Alemania ${ }^{6}$, resueltos de forma conjunta. La jurisprudencia fue elaborada en torno a la condicionalidad de la inmunidad de las organizaciones internacionales.

4. Se aprecia una noción subyacente del derecho al acceso a la justicia, implícitamente contenida en la prohibición de denegación de justicia del derecho internacional consuetudinario, como en instrumentos contemporáneos de derechos humanos como la Declaración General de los Derechos Humanos de Naciones Unidas, la Declaración de Americana de los Derechos y Deberes del Hombre, la Convención Americana de Derechos Humanos y la Convención Europea sobre Derechos Humanos (Reinisch, 2008).

5. Caso Waite \& Kennedy con Alemania, Tribunal Europeo de Derechos Humanos, rol 26.083/94, 18 de febrero de 1999.

6. Caso Beer \& Regan con Alemania, Tribunal Europeo de Derechos Humanos, rol 28.934/95, 18 febrero de 1999. 
En ambos casos, la cuestión era si el derecho de acceso a la jurisdicción, reconocido por el artículo 6.1 del Convenio Europeo de Derechos Humanos, resulta vulnerado por un Estado parte cuando sus tribunales nacionales se declaran incompetentes de conocer un asunto en el que una organización internacional como la Agencia Espacial Europea (ESA) es demandada, por gozar de inmunidad de jurisdicción, específicamente, en materia laboral.

Los demandantes alegaban tener derecho a que su causa, relativa a la existencia o inexistencia de un vínculo laboral con la Agencia Espacial Europea regulado por el derecho alemán, fuese oída de forma equitativa y pública por un tribunal, por lo cual Alemania habría violado el artículo 6.1 del Convenio, en atención a que sus tribunales habían rechazado pronunciarse sobre el fondo del asunto al haber reconocido la inmunidad de jurisdicción conferida a esta organización, por medio del convenio de creación de la ESA.

El Tribunal Europeo de Derechos Humanos no cuestionó que los asuntos relativos a inmunidad se encontraban dentro de su jurisdicción, y coincidió con el gobierno alemán en que las acciones procesales ejercitadas por los demandantes ante la jurisdicción laboral alemana incidían en la determinación de sus «derechos y obligaciones de carácter civil» (Reinisch, 1999: 935).

Ahora, la cuestión principal no era exactamente la validez de la inmunidad, sino saber si Alemania había incumplido o no el Convenio Europeo de Derechos Humanos al conferir inmunidad a la ESA. Resulta interesante analizar el contenido de la resolución sobre este punto, pues arroja argumentos que configurarían la existencia de un quid pro quo en la concesión de inmunidades de jurisdicción a una organización internacional. El Tribunal de Estrasburgo no condenó a Alemania por violación del artículo 6.1. En una decisión adoptada por unanimidad, los jueces de la Gran Sala invocaron, entre otros argumentos, que el derecho de acceso a la jurisdicción tutelado por dicho artículo no es un derecho absoluto y puede verse sujeto a limitaciones. Lo anterior se traduce en que los Estados parte gozan de un margen de apreciación propio a la hora de regular un derecho que por su misma naturaleza precisa de regulación estatal. Sin perjuicio de lo anterior, es al Tribunal a quien le compete verificar si, al regular el derecho de acceso a la jurisdicción, los Estados han respetado su contenido esencial. De modo contrario, una limitación a este derecho fundamental no será compatible con el artículo 6.1, si la limitación no persigue un fin legítimo. ${ }^{7}$

Así, y analizado el caso bajo la exigencia de este requisito, el Tribunal señaló que la atribución de privilegios e inmunidades a las organizaciones internacionales constituye un medio esencial para garantizar su adecuado funcionamiento, al contribuir a ponerlas a salvo de interferencias unilaterales de los gobiernos. La práctica de otorgarles inmunidad de jurisdicción en sus instrumentos constitutivos, o en acuerdos

7. Waite \& Kennedy con Alemania, párrafo 59. 
suplementarios, cuenta con numerosos precedentes y su fundamento es el interés en el buen funcionamiento de dichas organizaciones. Tal práctica ha ido ganando en importancia a medida que se ha ampliado y reforzado la cooperación internacional en todos los ámbitos de la sociedad moderna. A la vista de todo ello, la inmunidad de jurisdicción reconocida por los tribunales alemanes a la ESA no carecía de un fin legítimo. ${ }^{8}$

Luego, el Tribunal examinó el requisito de proporcionalidad, al afirmar que «un factor material a la hora de determinar si otorgar inmunidad a la ESA respecto de la jurisdicción alemana vulnera o no el Convenio es si los demandantes disponían de alternativas razonables para proteger de modo efectivo los derechos tutelados por el Convenio». ${ }^{9} \mathrm{Al}$ respecto, los jueces consideraron que los demandantes habrían podido y debido recurrir a la ESA Appeals Board, órgano que según las normas internas de la organización es independiente y tiene competencia para resolver conflictos entre la Agencia y un miembro de su personal. Según la Corte, dicho cuerpo debería haber dado respuesta a la cuestión de si los demandantes eran empleados o no de la organización..$^{10}$

Ahora bien, el Tribunal adoptó una posición clara de condicionalidad de las inmunidades concedidas a una organización internacional, lo cual además se traduce en una solución inter partes. De este modo, se considera que la sentencia en los asuntos Waite \& Kennedy y Beer \& Regan contienen alguna afirmación de principios que resulta del máximo interés para el problema de las inmunidades de las organizaciones internacionales, en particular cuando entran en contacto con la jurisdicción de un Estado, derivado de conflictos con terceros particulares. En este sentido, y una vez que el Tribunal de Estrasburgo se inclinó por rechazar la demanda presentada, formuló como suya la opinión de que

cuando unos Estados crean organizaciones internacionales para cooperar en determinadas esferas de ciertas competencias y les otorgan inmunidades, la protección de los derechos fundamentales puede resultar afectada. Ahora bien, sería incompatible con el objeto y fin del Convenio que los Estados parte quedaran así exonerados de toda responsabilidad en relación con el Convenio en la esfera de actividad objeto de esa atribución. ${ }^{11}$

8. Waite \& Kennedy con Alemania, párrafo 63.

9. Waite \& Kennedy con Alemania, párrafo 69.

10. El Tribunal de Estrasburgo planteó además como otra solución posible en el caso, que los demandantes demandaran por daños, ante la jurisdicción alemana, a las empresas que en un principio los contrataron y que luego los cedieron a la Agencia Espacial Europea. Waite \& Kennedy con Alemania, párrafo 70 .

11. Waite \& Kennedy con Alemania, párrafo 67. 
Hay otros casos posteriores que han sido abordados por el Tribunal Europeo de Derechos Humanos en este ámbito, que no podremos analizar por razones de espacio. ${ }^{12}$

\section{Algunas sentencias de tribunales nacionales}

Hay muchos casos en diversos países que no alcanzaremos a abordar en este artículo, por lo que mostraremos algunos, a título ejemplar, de países que han ido abriéndose a estas nuevas doctrinas.

\section{Duhalde con Organización Panamericana de Salud}

El primero es el caso Duhalde con Organización Panamericana de Salud (OPS), decidido por sentencia de la Corte Suprema de Justicia de la Nación (Argentina) en recurso extraordinario, el 31 de agosto de $1999 .{ }^{13}$

Duhalde, funcionario de la citada organización, presentó una demanda laboral contra la OPS. Como en todos los casos, la organización demandada solicitó que se rechace la demanda por la excepción de incompetencia del tribunal con sustento en el privilegio de inmunidad de jurisdicción.

En la sentencia, la Corte reconoció (admitió) la inmunidad de jurisdicción de la OPS basado en que Argentina fue parte de la Convención de Naciones Unidas sobre prerrogativas e inmunidades de sus organismos especializados, ${ }^{14}$ pero también reconoce que la OPS había demostrado que tiene amplios procedimientos administrativos dentro de su régimen interno para ventilar de manera adecuada reclamos que se deriven de la relación de empleo, lo que incluye acceso al Tribunal Administrativo de la OIT.

La ratio decidendi se encuentra en su considerando 10, en el que se afirma que el reconocimiento de la inmunidad de jurisdicción no es absoluta aunque se haya suscrito un tratado internacional en tal sentido, porque se supedita a las condiciones constitucionales y de derechos fundamentales envueltos del caso concreto, en particular, que se cumpla con la «necesidad de establecer procedimientos apropiados para la resolución de controversias a que den lugar los contratos u otras controversias de

12. Entre ellos, sentencia del caso Madres Stiching de Srebrenica y Otros con Países Bajos, Tribunal Europeo de Derechos Humanos, rol 65542/12, 11 junio 2013; y sentencia del caso Luis María López Cifuentes con España, TEDH 2009\75, 7 julio 2009.

13. Sentencia de Corte Suprema de Justicia de la Nación, 31 de agosto de 1999, disponible en base de datos Vlex, en http://vlex.com/vid/-39923959.

14. Resolución 179 de la Asamblea de Naciones Unidas sobre Prerrogativas e Inmunidades de los Organismos Especializados, de la que es parte la República Argentina, en virtud de su aprobación por el Decreto Ley 7.672, del 13 de septiembre de 1963. 
derecho privado en las cuales sea parte el organismo especializado», por lo que «no puede alegarse válidamente un supuesto de privación de justicia, ya que existe un procedimiento para la solución de controversias».

\section{Illemassene con OECD}

El 29 de septiembre de 2010, la Corte de Casación (Sala Social) de Francia decidió el caso Illemassene con OECD. ${ }^{15}$ Se trata de una demanda laboral en contra de una organización internacional, y si bien el demandante reconoce que existe un tribunal administrativo de la OCDE para resolver el litigio, acuso que éste no es ni imparcial ni independiente, por lo que se violó la concepción francesa de política pública internacional y el artículo 6 numeral 1 del Convenio Europeo de Derechos Humanos. El Tribunal desestimó la demanda, pues consideró que la OCDE provee un tribunal administrativo que entrega garantías suficientes al trabajador sobre su derecho de acceso al tribunal y a un proceso equitativo, en términos de que no «era contrario al diseño francés del orden público internacional».

\section{M.X. con Banque Africaine de Développement}

En el caso M.X. con Banque Africaine de Développement ${ }^{16}$ la Corte de Casación de Francia acogió una demanda en contra del Banco Africano de Desarrollo, a pesar de que el demandante había sido empleado por una organización internacional que opera en África, al tomar en cuenta la circunstancia de que dicho empleado poseía la nacionalidad francesa - lo que determina una conexión con Francia-, y en esos términos se encontraba protegido por el Convenio Europeo de Derechos Humanos, que también - obviamente- vincula al Estado francés. En este caso, la organización demandada no podía hacer uso de la inmunidad de jurisdicción, ya que en el momento de los hechos no había instituido un tribunal con jurisdicción para resolver disputas de la naturaleza presente en este proceso. Decidir lo contrario implicaría una denegación de justicia y obstaculizar el ejercicio de un derecho que se enmarca en la política pública internacional de Francia.

\section{Trabajadores con OEI}

En este acápite encontramos tres casos laborales resueltos por la Corte Suprema de Colombia, entre varios trabajadores en contra de la Secretaría Ejecutiva del Convenio Andrés Bello (SECAB) y la Organización de Estados Iberoamericanos (OEI). ${ }^{17}$

15. Corte de Casación (Sala Social) de Francia, rol 09-41030, 29 de septiembre de 2010.

16. Corte de Casación (Sala Social) de Francia, rol 04-41012, 25 de enero de 2005.

17. Casos Trabajadores con OEI 1, auto de Corte Suprema de Justicia de Colombia, sala de Casación 
En estos tres casos, la Corte señaló que no resulta aplicable la Convención de Viena sobre Relaciones Diplomáticas a las organizaciones internacionales, pues «no todas [...] detentan inmunidad de jurisdicción por derecho propio o en razón de las funciones de carácter permanente que desarrollen». La inmunidad no es endógena ni deriva del derecho internacional consuetudinario, dado que su estatuto - incluyendo su régimen de inmunidades y privilegios - viene de lo que consagran los tratados constitutivos, convenios o acuerdos de sede, con el alcance que sus miembros decidan. Ello no significa que los Estados puedan eliminar la inmunidad de una organización cuando hayan acordado concederla, si bien es cierto los Estados fijan y delimitan los alcances de la inmunidad.

Además, según diferentes convenios internacionales de derechos humanos, dicha exención procesal no puede privar al particular afectado del derecho al acceso a la justicia, razón por la que es indispensable que la organización cuente con mecanismos apropiados para la resolución de controversias suscitadas con sus trabajadores, bien sea a través de tribunales propios o jurisdicción arbitral o internacional con garantías suficientes. Ello, agrega,

ha encontrado respaldo en el derecho internacional de las organizaciones internacionales y la jurisprudencia extranjera y nacional, que ha considerado que la inmunidad de jurisdicción de las organizaciones internacionales encuentra un límite en el derecho a la justiciabilidad, puesto que la validez de dichas cláusulas que consagran la inmunidad a favor de estos organismos descansan en el correlativo establecimiento de mecanismos de justicia efectiva (quid pro quo).

Las sentencias ejemplifican con los tribunales administrativos establecidos en las principales organizaciones para dirimir conflictos laborales con sus funcionarios, como los tribunales Contencioso-Administrativo y de Apelaciones de Naciones Unidas, y el Tribunal Administrativo de la OIT. Otros mecanismos de «justicia efectiva» están constituidos por «los pactos de cláusulas compromisorias para la arbitración de los conflictos derivados de contratos de servicios, o el establecimiento de procedimientos especiales para la resolución de controversias». La Corte añade que la competencia de los tribunales nacionales nace únicamente cuando la organización internacional - «a pesar de gozar de inmunidad absoluta según el tratado constitutivo, convención o acuerdo sede»— no garantiza a sus trabajadores el acceso a instrumentos de justicia efectiva. Por tanto, corresponde al juez

establecer si la cláusula de inmunidad pactada a favor del ente internacional está acompañada de mecanismos adecuados y apropiados para el restablecimiento de los derechos de los trabajadores afectados, pues se insiste, en ningún caso, el acuerdo de

Laboral, proceso AL3295-2014, 9 de abril de 2014; Trabajadores con OEI 2, proceso 69710, 4 de marzo de 2015; Trabajadores con $\mathrm{OEI}$ 3, proceso 64054, 11 de marzo de 2015. 
inmunidad puede hacer declinar la justiciabilidad de una organización internacional en esta especial materia.

\section{A.A.C.B. con ONU}

En la sentencia del caso A.A.C.B. con ONU, Programa Mundial de Alimentos pronunciado por la Corte Suprema de Colombia, ${ }^{18}$ el tribunal nuevamente destaca que este caso no se trata de inmunidades jurisdiccionales propias de agentes diplomáticos, sino de organizaciones internacionales, que son de otra naturaleza, pues la inmunidad no surge de modo endógeno ni deriva del derecho internacional, sino que depende de sus Estados miembros «según lo establezcan los tratados constitutivos, convenios o acuerdos de sede». Con todo, la inmunidad de las organizaciones nunca puede entenderse como absoluta, pues

si bien es cierto éstos fijan y delimitan los alcances de la inmunidad de las organizaciones internacionales, también lo es que conforme a diferentes convenios internacionales de derechos humanos, dicho beneficio no puede privar al particular afectado del derecho al acceso a la justicia, razón por la que es indispensable que la organización cuente con mecanismos apropiados para la resolución de las controversias suscitadas con sus trabajadores, bien sea a través de tribunales propios o jurisdicción arbitral o internacional con garantías suficientes.

En este caso, la organización demandada proveyó al demandante de vías apropiadas de solución de disputas derivadas de su vínculo contractual, mediante arbitraje de la Comisión de las Naciones Unidas para el Derecho Mercantil Internacional (CNUDMI). Dado lo anterior, la Corte reconoció la inmunidad de jurisdicción de la organización.

\section{Rayo con UNODC-PNUD}

En la sentencia de la Corte Suprema de Colombia en Rayo con UNODC-PNUD, ${ }^{19}$ las consideraciones 3 y siguientes son semejantes a las de las sentencias descritas en los casos Trabajadores con OEI. La Corte añade que no existe una inmunidad absoluta respecto de las organizaciones internacionales, y que debe atenderse a los siguientes parámetros:

18. Auto de Corte Suprema de Justicia, Novena Sala de Casación Laboral, proceso AL3289-2014, 9 de abril de 2014 .

19. Sentencia del caso G.P.V. Rayo con Oficina de Naciones Unidas contra la Droga y el Delito (UNODC) y/o Programa de Naciones Unidas para el Desarrollo (PNUD), Corte Suprema de Justicia (Sala de Casación Laboral), rol T42300, 27 de enero de 2016 (acción de tutela para obtener el amparo de sus derechos constitucionales fundamentales). 
no todas las organizaciones internacionales detentan inmunidad de jurisdicción por derecho propio o en razón de las funciones de carácter permanente que desarrollen; [...] gozan o no de inmunidad de jurisdicción, según lo establezcan los tratados constitutivos, convenios o acuerdos de sede, según la voluntad de sus miembros; [...] los Estados no pueden eliminar en términos absolutos la justiciabilidad de una organización internacional, cuando convengan conceder el beneficio de la inmunidad absoluta, puesto que la validez de dichas cláusulas que la consagran tiene un límite en el correlativo establecimiento de mecanismos apropiados o instrumentos de justicia efectiva que garanticen los derechos de los trabajadores; [...] corresponde al juez laboral en cada caso establecer si la cláusula de inmunidad pactada a favor del ente internacional está acompañada de mecanismos adecuados para el restablecimiento de los derechos de los trabajadores afectados.

\section{Da Cunda Terra con Organización Panamericana de Salud}

En el caso Da Cunda Terra con Organización Panamericana de Salud, la Corte Suprema de Uruguay no estima suficiente que la organización internacional considere un Tribunal Administrativo para resolver las disputas respecto de un reclamo laboral. ${ }^{20}$ Afirma que las inmunidades jurisdiccionales deben considerarse como normas de carácter excepcional más aún respecto de las organizaciones internacionales, ya que son restringidas y, por lo tanto, la inmunidad solo aplica a los actos de naturaleza jure imperio, con excepción de consentimiento del Estado, transacciones mercantiles, contratos de trabajo, lesiones a las personas y daños a los bienes, propiedad, posesión y uso de bienes, propiedad intelectual e industrial, participación en sociedades u otras colectividades (artículos 10 a 15), como lo supone la Convención de Naciones Unidas sobre Inmunidades Jurisdiccionales de los Estados extranjeros de 2004, que si bien todavía no está en vigencia, contiene principios de derecho general internacional. Otra interpretación, además, implicaría la denegación de acceso a la justicia, que atentaría contra el principio de la igualdad entre los litigantes.

\section{Conclusiones}

Importantes juristas han sostenido nuevos criterios que fundamentan las inmunidades de jurisdicción de las organizaciones internacionales, en cierto tipo de casos, referidos a conflictos entre ellas y terceros particulares habitantes de los Estados anfitriones, que podemos denominar quid pro quo. Asimismo, estos criterios han sido sustentados de uno $\mathrm{u}$ otro modo por tribunales internacionales y nacionales al abocarse a esa clase de casos.

20. Da Cunda Terra con Organización Panamericana de Salud, Corte Suprema de Uruguay, ficha 2-30676/2004, Diario Oficial núm. 28.378, 26 de diciembre de 2011. 
Como conclusiones de lo expuesto, estos criterios dicen relación con los siguientes elementos:

- Los Tribunales internacionales deben entregar vías adecuadas, útiles, eficaces de solución de conflictos con quienes se encuentren en conflicto legal. Es decir, vías adecuadas según los criterios de los derechos de acceso a la justicia, al debido proceso y a la tutela judicial efectiva.

- Dicha obligación constituye una especie de «contraprestación» o quid pro quo frente a los Estados que le reconocen la inmunidad jurisdiccional.

- Dicha obligación se sustenta en el principio de buena fe que debe gobernar las relaciones entre los sujetos de derecho internacional.

- La obligación de quid pro quo también se ve en la obligación de respeto de los derechos humanos que mantienen los Estados y las organizaciones internacionales y de que gozan las personas, en el ámbito del derecho internacional.

- En caso de que las organizaciones no otorguen dichas vías de solución de conflicto, tribunales estatales deben hacerse cargo de resolverlo, sin considerar la inmunidad de jurisdicción para ese caso concreto.

- La doctrina de quid pro quo se sustenta en un ejercicio de balance de intereses y derechos que deben sopesarse en los casos concretos, entre los fundamentos por los que los Estados otorgan inmunidades de jurisdicción a las organizaciones internacionales, los derechos humanos de las personas y los criterios universales de derecho constitucional de los países en los que se encuentran los tribunales que deben resolver.

\section{Agradecimientos}

Este artículo fue presentado a la I Bienal Latinoamericana de Estudios sobre Derecho Internacional de los Derechos Humanos, en Santiago, Facultad de Derecho de la Universidad de Chile, el 28 y 29 de noviembre de 2018.

\section{Referencias}

AL JAGHoub, Mahasen M. (2006). The advisory function of the International Court of Justice 1946-2005. Berlín: Springer Heildelberg.

De Brabandere, Eric (2010). «Immunity of International Organizations in PostConflict International Administrations». International Organizations Law Review, 7 (1): 79-119,

Espósito, Carlos (2007). Inmunidad del Estado y derechos humanos. Madrid: Civitas. 
ReINIsCH, August (1999). «Waite and Kennedy v. Germany, application No. 26083/94; Beer and Regan v. Germany, application No. 28934/95». American Journal of International Law, 93 (4): 935-936. DOI: 10.2307/2555358.

-. (2008). «The immunity of international organizations and the jurisdiction of their Administrative Tribunals». Chinese Journal of International Law, 7 (2): 285-306.

\section{Sobre los autores}

Gloria Ramos-Fuentes es abogada de la Universidad de Chile. Magíster en Derecho Internacional de la Universidad Complutense de Madrid, España. Actualmente es doctoranda de la Universidad Autónoma de Chile y se desempeña como abogada de la Dirección Jurídica del Ministerio de Relaciones Exteriores de Chile. Su correo electrónico es gloramos76@hotmail.com.

Patricio Masbernat es abogado de la Pontificia Universidad Católica de Chile. Magíster y doctor en Derecho por la Universidad Complutense de Madrid, España, y se desempaña como profesor de la Universidad Autónoma de Chile. Su correo es patricio.masbernat@uautonoma.cl. 
